DOI: https://doi.org/10.46296/yc.v4i7edesp.0064

\title{
ENFOQUES PSICOLÓGICOS Y SUS PRINCIPIOS ÉTICOS
}

\section{PSYCHOLOGICAL APPROACHES AND THEIR ETHICAL PRINCIPLES}

\author{
Vera-García Blanca Maritza de los Ángeles ${ }^{1 *}$; Caicedo-Guale Liliana Carolina²; \\ Cedeño-Mejía Robert Gonzalo ${ }^{3}$; Hidalgo-Moreira Marcelo Alfonso ${ }^{4}$ \\ ${ }^{1}$ Docente de la Universidad Técnica de Manabí, UTM. Portoviejo, Ecuador. \\ ${ }^{2}$ Docente de la Universidad Laica Eloy Alfaro de Manabí, ULEAM. Manta, Ecuador. \\ 3, 4 Universidad Técnica de Manabí, UTM. Portoviejo, Ecuador.
}

*Correo: blanca.vera@utm.edu.ec

\begin{abstract}
Resumen
La psicología, a diferencia de otras profesiones, no tiene un criterio unificado de intervención sino una multiplicidad de enfoques que direccionan la actuación profesional. El papel de los principios éticos en el ejercicio profesional del psicólogo es una actividad que lo coloca en situaciones en las que se ve comprometido en la toma de decisiones, frente a circunstancias en las que se cuestiona su proceder profesional y las consecuencias de este. (Alfaro, 2012). Partiendo de esto, se han conformado organizaciones para regular el accionar de los profesionales de la salud mental, tal es el caso de la centenaria American Psychological Association (APA), la cual ya en 1938 creó su Comité de ética científica y profesional (Pope \& Vetter, 2016). A partir de la fundamentación de los enfoques éticos y cada principio, en esta investigación se pretenden identificar semejanzas y diferencias éticas de los enfoques psicológicos de mayor incidencia, y también relacionar los principios deontológicos propios de cada enfoque psicológico con los principios éticos propuestos por la APA.
\end{abstract}

Palabras clave: Psicología, APA, principios éticos.

\begin{abstract}
Psychology, unlike other professions, does not have a unified criterion of intervention but a multiplicity of approaches that guide professional performance. The role of ethical principles in the professional practice of the psychologist is an activity that places him in situations in which he is involved in decision-making, in the face of circumstances in which his professional behavior and the consequences of this are questioned. (Alfaro, 2012). Based on this, organizations have been formed to regulate the actions of mental health professionals, such is the case of the centennial American Psychological Association (APA), which already in 1938 created its Scientific and Professional Ethics Committee (Pope \& Vetter, 2016). Based on the foundation of the ethical approaches and each principle, this research aims to identify similarities and ethical differences of the psychological approaches of greater incidence, and to relate the deontological principles of each psychological approach with the ethical principles proposed by the APA.
\end{abstract}

Keywords: Psychology, APA, ethical principles.

Información del manuscrito:

Fecha de recepción: 14 de septiembre de 2020.

Fecha de aceptación: 12 de noviembre de 2020.

Fecha de publicación: 16 de noviembre de 2020. 


\section{Introducción}

El primer código ético de la APA surgió en 1953 con una característica que lo distinguía de otros códigos de sociedades científicas y profesionales. En efecto, dicho código se basó en una investigación empírica destinada a identificar los dilemas éticos frente a los que se enfrentaban los psicólogos (Hobbs,1948). Los aspectos indicados por los profesionales como conflictivos son los que dieron lugar al primer borrador de código ético, el cual, luego de varias discusiones y modificaciones, se transformaría en las primeras pautas éticas para el ejercicio de la profesión (American Psychological Association, 2003).

El año de creación del primer código, y más tarde la aparición de sus cinco principios básicos, no coincide históricamente con el surgimiento de las corrientes psicológicas detrás de los enfoques psicoterapéuticos de mayor incidencia; por tanto los principios deontológicos de estos enfoques no coinciden con los principios propuestos por la American Psychological Association (principio de Beneficencia y no Maleficencia, Fidelidad y
Responsabilidad, Integridad, Justicia, y Respeto por los derechos y la dignidad de las personas).

Cada enfoque representa un aporte al arsenal de herramientas de intervención del psicólogo en los diversos casos que le toca abordar. Las principales características de los enfoques clásicos se presentan a continuación:

El enfoque psicodinámico tiene como objeto de estudio e intervención al inconsciente y su finalidad terapéutica es hacer consciente lo que no es consciente. Utiliza técnicas como el insight que implica conectar hechos, relacionar experiencias, vincular actos con ciertas situaciones, siendo el mismo paciente quien descubre tales conexiones.

El enfoque cognitivo-conductual tiene como objeto de estudio la cognición, la emoción y la conducta y su finalidad terapéutica es instaurar pensamientos, emociones y conductas adaptativas. Hace uso de técnicas como el reforzamiento (refuerzos y castigo) que consiste en aplicar consecuencias positivas 0 
negativas que configuran la conducta de la persona.

El enfoque humanista-existencial, cuyo objeto de estudio es el ser humano en su totalidad y apunta terapéuticamente a la autorrealización, hace uso de técnicas respaldadas por la filosofía, especialmente la existencial; una de ellas es el relacionamiento de acompañamiento, propuesto por Rogers, consistente en ir junto al cliente en su proceso de crecimiento o recuperación, sin intervenir directivamente, sólo orientando desde el papel profesional.

El enfoque sistémico familiar, concreta su acción a las relaciones familiares y la comunicación y su finalidad es la funcionalidad familiar. Hace uso de técnicas como la redefinición que consiste en cambiar el sentido del síntoma presente en el grupo familiar.

El enfoque integrativo focaliza su actuación al ser humano desde su dimensión biológica, psicológica, social y espiritual). Su objetivo final es "Ayudar a que el paciente se ayude a sí mismo" (Opazo, 2001). Las técnicas que utiliza son procedentes de todos los enfoques, pero adaptadas según un supraparadigma.

El enfoque biologicista centra su accionar a los procesos anatómicos y fisiológicos que sustentan los procesos psicológicos; su finalidad es la funcionalidad psicofisiológica. Hace uso de técnicas propias de la neurorehabilitación y la farmacología.

Los principios, de los cuales se derivan las normativas que componen los diferentes países adscritos a la APA son los siguientes:

- Principio de Beneficencia y No Maleficencia: según la APA este principio consiste en que "los psicólogos se esfuerzan por hacer el bien a aquellos con quienes interactúan profesionalmente y asumen la responsabilidad de no hacer daño" (Facultad de Psicología, 2010).

- Principio de Fidelidad y Responsabilidad: de acuerdo con la APA consiste en que "los psicólogos establecen relaciones de confianza con aquellos con quienes trabajan. Son conscientes de sus 
responsabilidades profesionales y científicas con la sociedad y las comunidades específicas donde interactúan" (Facultad de Psicología, 2010).

- Principio de Integridad: para la APA en este principio "los psicólogos buscan promover la exactitud, honestidad y veracidad en la ciencia, docencia, y práctica de la psicología" (Facultad de Psicología, 2010).

- Principio de Justicia: en el sentido de la APA, este principio consiste en lo siguiente:

Los psicólogos reconocen que la imparcialidad y la justicia permiten que todas las personas accedan y se beneficien de los aportes de la psicología, y que se equipare la calidad de los procesos, procedimientos y servicios llevados a cabo por los psicólogos. (Facultad de Psicología, 2010)

- Principio de Respeto por los derechos y la dignidad de las personas: la APA en relación a este principio concibe que "los psicólogos respetan la dignidad y el valor de todas las personas y el derecho a la privacidad, a la confidencialidad y a la autodeterminación de los individuos" (Facultad de Psicología, 2010).

\section{Metodología}

La investigación que respalda este artículo es de enfoque cualitativo y de un nivel descriptivo ya que se caracterizó la realidad psico-ética en la que se han basado los diversos enfoques psicológicos; así mismo, es documental por la utilización de fuentes secundarias para recabar información electrónica. Una vez lograda la obtención de la información necesaria, ésta se sometió a un análisis comparativo en cuadros de doble entrada, aplicadas a los enfoques predominantes (Psicodinámica, Cognitivo conductual, Humanista-Existencial, Sistémica, Integrativa, Biologista). Los criterios correspondientes a los enfoques fueron: Objeto de estudio, Finalidad de la intervención, Relación objeto - sujeto (terapéutico) y Principios deontológicos. Posterior a este análisis se buscaron los puntos en común en los aspectos éticos inherentes a cada enfoque psicológico. 


\section{Resultados}

Tabla 1. Resultados del trabajo de investigación.

\begin{tabular}{|c|c|c|c|c|c|c|}
\hline & Psicodinámica & $\begin{array}{l}\text { Cognitivo - } \\
\text { conductual }\end{array}$ & $\begin{array}{l}\text { Humanista - } \\
\text { existencial }\end{array}$ & $\begin{array}{l}\text { Sistémico } \\
\text { Familiar }\end{array}$ & Integrativo & Biologicista \\
\hline $\begin{array}{l}\text { Objeto de } \\
\text { estudio }\end{array}$ & El inconsciente & $\begin{array}{l}\text { Cognición, } \\
\text { emoción } \\
\text { conducta }\end{array}$ & $\begin{array}{l}\text { El ser humano en su } \\
\text { totalidad }\end{array}$ & $\begin{array}{l}\text { Relaciones } \\
\text { familiares } \\
\text { comunicación }\end{array}$ & $\begin{array}{l}\text { El ser humano } \\
\text { (biológico, } \\
\text { psicológico, } \\
\text { social } \\
\text { espiritual) }\end{array}$ & $\begin{array}{l}\text { Procesos } \\
\text { psicológicos y } \\
\text { sus bases } \\
\text { anatomo- } \\
\text { fisiológicas. }\end{array}$ \\
\hline $\begin{array}{l}\text { Finalidad de la } \\
\text { intervención }\end{array}$ & $\begin{array}{l}\text { Hacer } \\
\text { consciente lo } \\
\text { inconsciente }\end{array}$ & $\begin{array}{l}\text { Pensamiento, } \\
\text { emociones y } \\
\text { conductas } \\
\text { adaptativas }\end{array}$ & Autorrealización & $\begin{array}{l}\text { Funcionalidad } \\
\text { familiar }\end{array}$ & $\begin{array}{l}\text { "Ayudar a que el } \\
\text { paciente } \\
\text { ayude se a sí } \\
\text { mismo" (Opazo, } \\
\text { 2001) }\end{array}$ & $\begin{array}{l}\text { Funcionalidad } \\
\text { anatómica y } \\
\text { fisiológica }\end{array}$ \\
\hline Técnicas & $\begin{array}{l}\text {-Insight } \\
\text {-Experiencia } \\
\text { Emocional } \\
\text { Correctiva } \\
\text {-Asociación libre } \\
\text {-Interpretación } \\
\text { de los sueños } \\
\text {-Análisis } \\
\text { transferencial y } \\
\text { contratransferen } \\
\text { cial. } \\
\text { (Fernández, } \\
\text { 2011) }\end{array}$ & $\begin{array}{l}\text {-Analisis } \\
\text { funcional } \\
\text {-Técnicas de } \\
\text { Exposición } \\
\text {-Refuerzos y } \\
\text { Castigo } \\
\text {-Modelado } \\
\text {-Reestructuració } \\
\text { n cognitiva } \\
\text {-Manejo de } \\
\text { contingencias. } \\
\text { (Fernández, } \\
\text { 2011) }\end{array}$ & $\begin{array}{l}\text {-Relacionamiento de } \\
\text { acompañamiento } \\
\text {-Logoterapia } \\
\text { (Martorell, 2014) } \\
\circ \quad \text { Cuestionamie } \\
\text { nto } \\
\circ \quad \text { De-reflexión } \\
\circ \quad \text { La intención } \\
\text { paradójica } \\
\circ \quad \text { Biblioterapia }\end{array}$ & $\begin{array}{l}\text {-Familiogramas } \\
\text {-Cambio de roles } \\
\text {-Redefinicioes } \\
\text {-Tareas } \\
\text {-Acomodación } \\
\text {-Restructuración } \\
\text {-Hipótesis } \\
\text {-Circularidad }\end{array}$ & $\begin{array}{l}\text {-Técnicas } \\
\text { psicodinámicas } \\
\text {-Técnicas } \\
\text { gestálticas } \\
\text {-Técnicas } \\
\text { cognitivas } \\
\text {-Técnicas } \\
\text { conductuales } \\
\text {-Farmacoterapia }\end{array}$ & $\begin{array}{l}\text {-Neurorehabilit } \\
\text { ación } \\
\text {-Farmacoterap } \\
\text { ia }\end{array}$ \\
\hline
\end{tabular}

\begin{tabular}{|c|c|c|c|c|c|}
\hline $\begin{array}{c}\text { Relación } \\
\text { objeto - } \\
\text { sujeto } \\
\text { (terapéutico) }\end{array}$ & No directiva & Directiva & Acompañamiento & Equidistante & $\begin{array}{c}\text { Adaptada al caso } \\
\text { (directiva, no } \\
\text { directiva, } \\
\text { acompañamiento) }\end{array}$ \\
\hline
\end{tabular}

DirectivaPrescriptiva

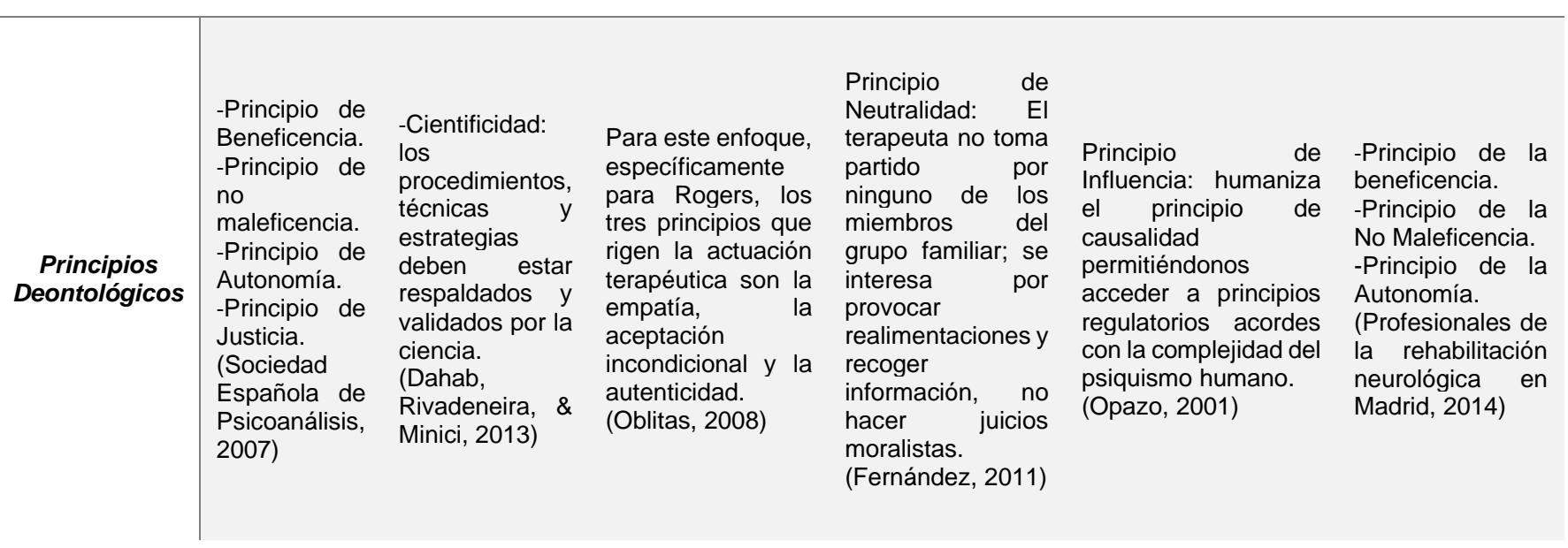

Fuente: Revisión documental. Elaborado por: Grupo de investigadores. 


\section{Discusión}

Históricamente las corrientes que sustentan los enfoques sometidos a análisis no coincidieron cronológicamente con el surgimiento de los principios éticos propuestos por la APA. Por tal razón, cada enfoque ha ido proponiendo gradualmente principios que rigen la actuación del profesional, desde el prisma teórico elegido, que en algunos casos no se homologan a los principios éticos de la Asociación de Psicología Americana.

En el enfoque psicodinámico, la actuación está patrocinada por cuatro principios, que se encuentran presentes en las intervenciones médicas también: principio de Beneficencia, de No Maleficencia, de Autonomía y de Justicia (Sociedad Española de Psicoanálisis, 2007). Principios que se encuentran también en el "supra-código" ético de la APA.

La intervención en el enfoque cognitivo conductual se encuentra regida sobre todo por el principio de la cientificidad; este hace imperante la aplicación de métodos y técnicas validadas científica y empíricamente; este principio compagina con el principio de integridad de la APA.

En el enfoque humanista-existencial la actuación terapéutica está amparada por los principios propuestos por Rogers (empatía, aceptación incondicional y autenticidad) concordantes con el principio de Respeto a los derechos y dignidad de las personas perteneciente a la APA.

El principio que preside la intervención del enfoque sistémico familiar es el de neutralidad, el cual consiste en que "el terapeuta no toma partido por ninguno de los miembros del grupo familiar; se interesa por provocar realimentaciones y recoger información, no hacer juicios moralistas" (Fernández, 2011); este principio concuerda con el principio de Justicia planteado por la APA.

El principio que rige el enfoque integrativo es el principio de influencia el cual "humaniza el principio de causalidad permitiéndonos acceder a principios regulatorios acordes con la complejidad del psiquismo humano" (Opazo, 2001.). Este principio no concuerda con ninguno de los 
principios propuestos por la APA y, no obstante, esto no le resta a su aspecto ético ya que al "humanizar" las explicaciones causales, su intervención no se da de un modo mecánico sino de una forma profundamente empática y a todos los niveles.

En el enfoque biologicista, los principios que gobiernan su intervención son los mismos que ejercen rectoría en las ramas médicas: principio de beneficencia, de No Maleficencia y de Autonomía, principios pertenecientes también a la APA.

\section{Conclusiones}

Los enfoques psicológicos mencionados son de gran importancia para la comunidad, ya que nos permite coadyuvar a la intervención específica desde cada concepción teórica, utilizando la que más se adapte a la situación terapéutica. A su vez, cada modelo difiere de los otros, pero comparten la misma finalidad: el mejor funcionamiento psicológico posible del ser humano, desde su propio objeto de estudio e intervención y con sus propias técnicas, primando su dignidad y bienestar por encima de cualquier aspecto.

Por otra parte, en la intervención psicoterapéutica, las prescripciones de las acciones a realizar por el profesional no sólo serán de índole teórica, sino también de naturaleza ética. Los psicólogos enfrentan dilemas éticos a lo largo de su profesión y es su deber hacerles frente, en función de los principios que propone la Asociación Americana de Psicología y los valores que establecen las escuelas psicológicas a las que adscriben.

Los principios nacientes en cada corriente no discrepan mucho de los planteados por la APA; todos ellos priorizan el bienestar de la persona en las intervenciones que realizan.

\section{Bibliografía}

Alfaro, K. (2012). Ética y Psicología. México: Red Tercer Milenio. ISBN 978-607-733-079-0.

American Psychological Association (2003). The first code. American Psychological Association, 34 (1), p. 63. Recuperado de: https://www.apa.org/monitor/j an03/firstcode.aspx 
Dahab, J., Rivadeneira, C., \& Minici, A. (2013). Las complicaciones de los abordajes indirectos en Psicología. Revista de Terapia Cognitiva Conductual, 1(22). Retrieved from

http://cetecic.com.ar/revista/la s-complicaciones-de-losabordajes-indirectos-enpsicologia/

Facultad de Psicología. (2010). Principios Éticos de los Psicólogos y Código de Conducta American Psychological Association (APA) Enmiendas 2010. Buenos Aires, Argentina. Recuperado de: http://www.psicologia.unam.m $\mathrm{x} /$ documentos/pdf/comite_etic a/Codigo_APA.pdf

Fernández, H. (2011). Paisajes de la Psicoterapia. Buenos Aires, Argentina: Polemos.

Martorell, J. L. (2014). Psicoterapias Escuelas y conceptos básicos. Editorial Pirámide.

Oblitas, L. (2008). Psicoterapias Contemporáneas. Santa Fe: Cengage Learning Editores S.A.

Opazo, R. (2001). Psicoterapia Integrativa Delimitación Clínica. Santiago de Chile: Instituto Chileno de Psicoterapia Integrativa.

Pope, K. y Vetter, V. (2016). Ethical Dilemmas Encountered by
Members of the American Psychological Association: A National Survey. ResearchGate. Recuperado de:

https://www.researchgate.net/ publication/11708579_Ethical _Dilemmas_Encountered_by _Members_of_the_American _Psychological_Association_ A_National_Survey

Profesionales de la rehabilitación neurológica en Madrid. (2014). Ética en rehabilitación neurológica ¿QUÉ DEBO HACER? Retrieved from https://rhbneuromad.wordpre ss.com/2014/07/18/etica-enrehabilitacion-neurologicaque-debo-hacer/

Sociedad Española de Psicoanálisis. (2007). Código ÉticoDeontológico. Retrieved from http://www.seppsicoanalisi.org/wpcontent/uploads/2016/12/Cod igo-Etico-SEP-2007.pdf 